Ann. Sci. forest., 1980, 37 (4), 275-277.

\title{
Le dépérissement du hêtre au Grand-Duché de Luxembourg
}

\author{
E. LIES \\ Ingénieur Principal des Eaux ef Forêts \\ 16, rue Rochefort-Luxembourg.
}

Le hêtre constitue l'essence la plus répandue au Grand-Duché de Luxembourg. Ainsi plus de la moitié de la forêt luxembourgeoise se compose de hêtres. Longtemps cette précieuse essence ne posait pas de problèmes du point de vue sanitaire.

Certes, le dépérissement du hêtre existe au Grand-Duché depuis des dizaines d'années déjà. Mais, il ne s'agissait en général que de quelques sujets dispersés qui venaient à sécher ef dont le repérage et l'exploitation s'avéraient particulièrement onéreux.

Ce fut en 1976 que la maladie est passée au stade de l'épidémie avec une intensité variable selon les régions. Ainsi, l'Oesling, la partie luxembourgeoise des Ardennes (altiłude 400-600 m NN) a été épargnée de façon générale, mais le reste du pays (et surtout la région mosellane) a été touché gravement.

Pour cetle partie du Grand-Duché où la maladie s'est manifestée essentiellement, les données écologiques sont les suivantes :

avril-septembre

Pluviosité

Température moyenne... Altitude ...............

Géologie...............

Sols.
$700-850 \mathrm{~mm}$

$9 \circ \mathrm{C}$

200-400 m NN

systèmes jurassique et triasique

sableux à marneux

Quelles ont été les conséquences de cette maladie?

Et bien, les coupes ont dû être concentrées dans les peuplements attaqués et le volume abattu à la suite du dépérissement du hêtre a pris des proportions impres. sionnantes.

Ainsi, il s'est élevé :

- en 1977 à $15000 \mathrm{~m}^{3}$ soit 10 p. 100 de la coupe annuelle tolale,

- en 1978 à $45000 \mathrm{~m}^{3}$ soit 25 p. 100 de la coupe annuelle totale, pour retomber en 1979 à $25000 \mathrm{~m}^{3}$ soit 15 p. 100 de la coupe annuelle totale.

Exprimés par rapport au volume exploité en hêtre, ces chiffres s'élèvent resp. à 20,50 et 30 p. 100 de la coupe totale en bois de hêtre. 
Pour le moment, il semble que la situation soit devenue moins alarmante étant donné que les attaques toujours actuelles sont de moindre envergure ef plus dispersées.

Mais les interventions parfois excessives ont laissé leurs traces. Par endroił les coupes sanitaires nous ont obligé à exploiter un nombre d'arbres anormalement élevé de sorte que la gestion normale de ces peuplements n'est plus assurée ef qu'il faut procéder à une régénération naturelle ou artificielle avant le terme d'exploitabilité.

Souvent la qualité des bois abattus, surtout des individus gravement atteints, ne permet plus leur usage comme bois de sciage. Ils doivent être cédés comme bois de trituration avec une moins-value certaine.

\section{Quels sont maintenant les peuplements les plus éprouvés?}

Si la maladie sévit un peu partout, on peut distinguer deux types de forêts particulièrement touchées :

- les peuplements mélangés de hêtre et de chêne croissant sur les marnes du système jurassique (Lias). Dans des conditions normales, la croissance du hêtre dépasse sur ces stations celle du chêne de sorte que le chêne disparaît s'il n'est pas largement favorisé lors des éclaircies. Faute d'interventions en faveur du chêne, le hêtre finit par dominer largement et en cas de maladie une telle forêt risque d'être anéantie complètement. Ceci vauł également pour le type de forêt suivant ;

- les peuplements mélangés de chêne, charme et hêtre croissant sur les marnes du système triasique (Keuper, Muschelkalk). Dans ces peuplements la part du hêtre est normalement moins importante (sol souvent mal drainé) de sorte que le nombre de chênes ef de charmes est presque toujours suffisant pour assurer le maintien du peuplement même en cas de dépérissement du hêtre.

Si la maladie semble apparaître sur toutes les formations géologique et pédologique du pays, une certaine concentration a pu être constatée sur les sols lourds, mais également sur les sols légers superficiels ( $p$. ex. où la roche affleure), donc sur des sols où l'approvisionnement en eau n'était pas assuré lors des années sèches telles que les années 1975 et 1976.

Il en est de même des expositions. Si la maladie se manifeste sur toutes les expositions, ce sont surtout les expositions chaudes (el sèches) qui sont les plus touchées.

Sont également gravement touchés les peuplements qui ont subi une insolation massive à la suite d'une percée ( $p$. ex. traversée d'une grande route, conduite d'eau ou d'énergie) ou à la suite de la mise à blanc du peuplement adjacent.

Quant à l'âge des peuplements touchés, il faut reconnaître que tous les stades sont touchés (du perchis au vieux bois). Certes, la plupart des foyers ont été constatés dans les vieux peuplements. Mais, il faut savoir que la très grande majorité de nos peuplements de hêtre sont âgés de 100 à 150 ans. Donc forcément la plupart des peuplements touchés figurent dans ces classes d'âge. Ce qu'on peut dire, c'est que dans les peuplements âgés les foyers sont parfois très étendus alors que dans les jeunes peuplements il ne s'agit en général que de quelques individus isolés. Ce qui également peuł être grave s'il s'agit des arbres d'élite. Quant aux jeunes peuplements touchés, il faut ajouter qu'il s'agit surtout de peuplements à l'état très serré. 
Que faut-il faire pour venir à bout de ceffe maladie?

Certainement il faut procéder aux coupes sanitaires. Faut-il également éliminer régulièrement les rémanents de ces coupes pour réduire les foyers de contamination à partir desquels se propage la pourriture blanche? Par ailleurs il ne nous reste qu'à espérer que les recherches aboutissent rapidement à mieux connaître l'étiologie de cette maladie afin de pouvoir mettre en ouvre une lutte chimique ou biologique appropriée et économiquement supportable.

Enfin ne faut-il pas tâcher à mieux connaître l'écologie du hêtre, ses exigences climatiques et stationnelles, voire repenser son traitement sylvicole? 